\title{
On the Lipschitz's condition for Brownian motion.
}

\author{
By K. L. ChunG*, P. Erdös and T. Sirao
}

(Received April 9, 1959)

(Revised May 25, 1959)

Let $X(t)(0 \leqq t<\infty)$ be the Brownian motion process. Concerning the uniform continuity of $X(t)$, there exists P. Lévy's result. Before stating his result, let us define the concept of upper class and lower class with regard to the uniform continuity of $X(t)(0 \leqq t \leqq 1)$.

If there exists a positive number $\varepsilon$ such that $\left|t^{\prime}-t\right| \leqq \varepsilon$ implies the relation

$$
\left|f\left(t^{\prime}\right)-f(t)\right| \leqq g\left(\left|t^{\prime}-t\right|\right),
$$

where $g(t)$ is a non-negative, continuous, non-decreasing function defined in some finite interval $(0, T)$ and vanishing with $t$, then we say that $f(t)$ satisfies Lipschitz's condition relative to $g(t)$. Putting $\varphi(t)=\psi\left(\frac{1}{t}\right) \sqrt{t}$, if $X(t)(0 \leqq t$ $\leqq 1$ ) satisfies Lipschitz's condition relative to $\varphi(t)$ with probability 1 we say that $\psi(t)$ belongs to the upper class. If $X(t)(0 \leqq t \leqq 1)$ does not satisfy Lipschitz's condition relative to $\varphi(t)$ with probability 1 we say that $\psi(t)$ belongs to the lower class. P. Lévy [1] proved that the function

$$
\psi(t)=c(2 \log t)^{\frac{1}{2}}
$$

belongs to the upper class for $c>1$ and belongs to the lower class for $c<1$. Following his method, T. Sirao [2] improved the result as follows: The function

$$
\psi(t)=(2 \log t+c \log \log t)^{\frac{1}{2}}
$$

belongs to the upper class for $c>5$ and belongs to the lower class for $c<-1$. In this paper we shall prove the following theorems.

THEOREM 1. A non-negative, continuous and monotone non-decreasing function $\psi(t)$ belongs to the upper or lower class according as the integral

$$
\int^{\infty} \psi^{3}(t) e^{-\frac{1}{2} \psi^{2}(t)} d t
$$

is convergent or divergent.

\footnotetext{
* Research supported in part by the United States Air Force through the Office of Scientific Research under contract AF 49 (638)-265.
} 
TheOREM 2. The function $\psi(t)$ defined by

$$
\psi(t)=\left(2 \log t+5 \log _{(2)} t+2 \log _{(3)} t+\cdots+2 \log _{(n-1)} t+c \log _{(n)} t\right)^{\frac{1}{2}},
$$

where $\log _{(n)} t$ denotes the $n$-times iterated logarithm, belongs to the upper class for $c>2$ and to the lower class for $c \leqq 2$.

These theorems were quoted by P. Lévy [3] without proof. They give a difinitive solution to the problem of uniform continuity of Brownian motion $X(t)$ and are comparable to A. Kolmogorov's criterion in the theory of iterated logarithm for $X(t)$ at time point $\infty$.

Theorem 2 is a simple corollary of Theorem 1. Hence we prove only Theorem 1.

Lemma 1. Without loss of generality, we may assume that

$$
(2 \log t-10 \log \log t)^{\frac{1}{2}} \leqq \psi(t) \leqq(2 \log t+10 \log \log t)^{\frac{1}{2}}
$$

Proof. We show that if Theorem 1 holds under the assumption (4), then it holds without (4). Let us denotes the first member in (4) by $\psi_{1}(t)$ and the last member in (4) by $\psi_{2}(t)$.

Define $\hat{\psi}(t)$ as follows :

$$
\hat{\psi}(t)=\min \left(\max \left(\psi(t), \psi_{1}(t)\right), \psi_{2}(t)\right) .
$$

Then the convergence of the integral (2) for $\psi(t)$ implies the same for $\hat{\psi}(t)$. In fact, let us assume the convergence of (2) for $\psi(t)$. If the set of $t$ on which $\psi(t)$ is less than $\psi_{1}(t)$ is not bounded, there exists an increasing sequence $\left\{t_{n}\right\}$ such that $\psi\left(t_{n}\right) \leqq \psi_{1}\left(t_{n}\right)$ and $t_{n}$ tends to infinity with $n$. Since $\psi(t)$ is a non-negative and non-decreasing function, we have

$$
\begin{aligned}
\int_{t_{1}}^{\infty} \psi^{3}(t) e^{-\frac{1}{2} \psi^{2}(t)} d t & \geqq \int_{t_{1}}^{t_{n}} \psi^{3}(t) e^{-\frac{1}{2} \psi^{2}(t)} d t \\
& \geqq c \psi^{3}\left(t_{n}\right) e^{-\frac{1}{2} \psi^{2}\left(t_{n}\right)} t_{n} \\
& \geqq c\left(\log t_{n}\right)^{\frac{13}{2}}
\end{aligned}
$$

where $c$ is a positive constant. Since $\log t_{n}$ tends to infinity with $n$, the integral for $\psi(t)$ is divergent. This contradicts our assumption and therefore $\psi_{1}(t)$ must be smaller than $\psi(t)$ for large $t$. On the other hand the integral for $\psi_{2}(t)$ is convergent. These facts prove our assertion. Now we assume that the integral for $\psi(t)$ is convergent and Theorem 1 valid under the condition (4). Then the integral for $\hat{\psi}(t)$ is convergent and therefore $\hat{\psi}(t)$ belongs to the upper class. But by what has just been shown $\hat{\psi}(t) \leqq \psi(t)$ for large $t$. So we have $\hat{\varphi}(h) \leqq \varphi(h)$ for small $h$ where $\hat{\varphi}(t)$ is defined by $\hat{\psi}(t)$ as $\varphi(t)$ is by $\psi(t)$ and therefore $\psi(t)$ belongs to the upper class. Thus Lemma 1 is proved in the convergent case. 
Secondly let us assume that the integral for $\psi(t)$ is divergent. If the set of $t$ on which $\psi(t)$ is less than $\psi_{1}(t)$ is bounded, then it follows that $\hat{\psi}(t)$ is less than $\psi(t)$ for large $t$ and accordingly the integral for $\hat{\psi}(t)$ must be divergent. On the contrary, if there exists an increasing sequence $\left\{t_{n}\right\}$ having the property

$$
\psi\left(t_{n}\right)<\psi_{1}\left(t_{n}\right), \quad t_{n} \rightarrow \infty \text { as } n \rightarrow \infty,
$$

then we have

$$
\hat{\psi}\left(t_{n}\right)=\psi_{1}\left(t_{n}\right) .
$$

By the monotony of $\hat{\psi}(t)$, we have

$$
\begin{aligned}
\int_{t_{1}}^{t_{n}} \hat{\psi}^{3}(t) e^{-\frac{1}{2} \hat{\psi}^{3}(t)} d t & \geqq \psi^{3}\left(t_{n}\right) e^{-\frac{1}{2} \hat{\psi}^{2}\left(t_{n}\right)\left(t_{n}-t_{1}\right)} \\
& =\psi_{1}^{3}\left(t_{n}\right) e^{-\frac{1}{2} \psi_{1}^{2}\left(t_{n}\right)}\left(t_{n}-t_{1}\right) .
\end{aligned}
$$

Since the last term in (8) tends to infinity with $n$, the integral for $\hat{\psi}(t)$ is divergent in our case. Now, by the result in [2], $\psi_{2}(t)$ belongs to the upper class and therefore, for almost all sample point $\omega$, there exists $\varepsilon$ such that

$$
\left|X\left(t^{\prime}, \omega\right)-X(t, \omega)\right|<\varphi_{2}\left(\left|t^{\prime}-t\right|\right) \text { for }\left|t^{\prime}-t\right|<\varepsilon,
$$

where $\varphi_{2}(t)$ is defined by $\psi_{2}(t)$ in the same way as $\varphi(t)$ is by $\psi(t)$. On the other hand, since by assumption $\psi_{2}(t)$ belongs to the lower class, for almost all $\omega$ we can choose a sequence $\left\{\left(t_{n}, t_{n}{ }^{\prime}\right)\right\}$ having the following properties

$$
\begin{gathered}
\left|X\left(t_{n}{ }^{\prime}\right)-X\left(t_{n}\right)\right|>\hat{\varphi}\left(\left|t_{n}{ }^{\prime}-t_{n}\right|\right), \\
\left|t_{n}{ }^{\prime}-t_{n}\right| \rightarrow 0 \quad \text { as } n \rightarrow \infty .
\end{gathered}
$$

From (9) and (10), we have

$$
\hat{\varphi}\left(\left|t_{n}^{\prime}-t_{n}\right|\right)<\varphi_{2}\left(\left|t_{n}^{\prime}-t_{n}\right|\right) .
$$

(11) shows that $\varphi(t)$ is at last equal to $\varphi(t)$ at $t=\left|t_{n}{ }^{\prime}-t_{n}\right|$. This fact and (10) show that $\psi(t)$ belongs to the lower class.

Q.E.D.

We now proceed to prove Theorem 1 .

1) Proof of the convergent case.

First of all we remark that it suffices to prove, for almost all $\omega$, the existence of a positive $\varepsilon^{\prime}$ such that

$$
X\left(t^{\prime}, \omega\right)-X(t, \omega) \leqq \varphi\left(\left|t^{\prime}-t\right|\right) \quad \text { for } \quad\left|t^{\prime}-t\right|<\varepsilon^{\prime} .
$$

In fact, let us assume that this assertion holds. Then it follows from the symmetry of Brownian motion that the probability of the existence of a positive $\varepsilon^{\prime \prime}$ satisfying the inequality

$$
-\varphi\left(\left|t^{\prime}-t\right|\right) \leqq X\left(t^{\prime}, \omega\right)-X(t, \omega) \quad \text { for } \quad\left|t^{\prime}-t\right|<\varepsilon^{\prime \prime}
$$


is equal to 1 . Taking $\varepsilon$ for the minimum of $\varepsilon^{\prime}$ and $\varepsilon^{\prime \prime}$, we have Theorem 1 . Therefore we may consider the difference $X\left(t^{\prime}\right)-X(t)$ instead of its absolute value.

For each triple $(p, k, l)$, let $E_{k, l}^{p}$ be the event

$$
\begin{aligned}
X\left(\frac{k+l}{2^{p}}\right)-X\left(\frac{k}{2^{p}}\right) \geqq \varphi\left(\frac{l}{2^{p}}\right), \quad k & =0,1,2, \cdots, 2^{p}, \\
l & =1,2, \cdots, p .
\end{aligned}
$$

A simple computation shows that

$$
P\left(E_{k, l}^{p}\right) \sim e^{-\frac{1}{2} \psi^{2}\left(\frac{2^{p}}{l}\right)} /(2 \pi)^{\frac{1}{2}} \psi\left(\frac{2^{p}}{l}\right)
$$

for large $p$. Summing up $P\left(E_{k, l}^{p}\right)$ for $p=1,2, \cdots, \quad k=1,2, \cdots, 2^{p}, l=\left[\frac{p}{3}\right]$, $\left[\frac{p}{3}\right]+1, \cdots, p$, we have

$$
\begin{aligned}
\sum_{p=1}^{\infty} \sum_{k=1}^{2^{p}} \sum_{l=\left[\begin{array}{l}
p \\
3
\end{array}\right]}^{p} P\left(E_{k, l}^{p}\right) & =O(1) \sum_{p=1}^{\infty} \sum_{k=1}^{2^{p}} \sum_{l=\left[\begin{array}{l}
p \\
3
\end{array}\right]}^{p} e^{-\frac{1}{2} \psi^{2}\left(\begin{array}{c}
2^{p} \\
l
\end{array}\right)} / \psi\left(\frac{2^{p}}{l}\right) \\
& =O(1) \sum_{p=1}^{\infty} \sum_{k=1}^{2^{p}} \frac{p}{\psi\left(\frac{2^{p}}{p}\right)} e^{-\frac{1}{2} \psi^{2}\left(\frac{2^{p}}{p}\right)} .
\end{aligned}
$$

Applying Lemma 1, we obtain

$$
\begin{aligned}
\sum_{p=1}^{\infty} \sum_{k=1}^{2^{p}} \sum_{l=\left[\frac{p}{3}\right]}^{p} P\left(E_{k, l}^{p}\right) & =O(1) \sum_{p=1}^{\infty} \frac{2^{p}}{p} \psi^{3}\left(\frac{2^{p}}{p}\right) e^{-\frac{1}{2} \psi^{2}\left(\frac{2^{p}}{p}\right)} \\
& =O(1) \int^{\infty} \psi^{3}(t) e^{-\frac{1}{2} \psi^{2}(t)} d t<+\infty
\end{aligned}
$$

Next, for each triple $(p, k, l)$, let $F_{k, l}^{p}$ be the event

$$
\begin{aligned}
\max _{0 \leqq t, s \leqq \frac{1}{2^{p}}}\left\{X\left(\frac{k+l}{2^{p}}+t\right)-X\left(\frac{k}{2^{p}}-s\right)\right\} & \geqq \sqrt{\frac{l}{2^{p}}} \psi\left(\frac{2^{p}}{l+2}\right), \\
k & =0,1,2, \cdots, 2^{p}, \\
l & =1,2, \cdots, p .
\end{aligned}
$$

For convenience' sake, we consider the $F_{k, l}^{p}$ only such that the time parameters $t$ of $X(t)$ which appear in the above definition are positive and less than 1 . It is well known that

$$
P\left(\max _{0 \leqq s \leqq t} X(s)>a\right) \leqq 2 P(X(t)>a),
$$

where $a$ is any real number. Since the Brownian motion is an additive process, we have 
(15)

$$
\begin{array}{r}
P\left(F_{k, l}^{p}\right) \leqq P\left\{\max _{0 \leqq t \leqq \frac{1}{2^{p}}}\left(X\left(\frac{k+l}{2^{p}}+t\right)-X\left(\frac{k+l}{2^{p}}\right)\right)+\left(X\left(\frac{k+l}{2^{p}}\right)-X\left(\frac{k}{2^{p}}\right)\right)\right. \\
\left.\quad+\max _{0 \leqq s \leqq \frac{1}{2^{p}}}\left(X\left(\frac{k}{2^{p}}\right)-X\left(\frac{k}{2^{p}}-s\right)\right) \geqq \sqrt{\frac{l}{2^{p}}} \psi\left(\frac{2^{p}}{l+2}\right)\right\} \\
\leqq 4 P\left\{X\left(\frac{k+l+1}{2^{p}}\right)-X\left(\frac{k+l}{2^{p}}\right)+X\left(\frac{k+l}{2^{p}}\right)-X\left(\frac{k}{2^{p}}\right)\right. \\
\left.\quad+X\left(\frac{k}{2^{p}}\right)-X\left(\frac{k-1}{2^{p}}\right) \geqq \sqrt{\frac{l}{2^{p}}} \psi\left(\frac{2^{p}}{l+2}\right)\right\} \\
=4 P\left(X\left(\frac{k+l+1}{2^{p}}\right)-X\left(\frac{k-1}{2^{p}}\right) \geqq \sqrt{\frac{l}{2^{p}}} \psi\left(\frac{2^{p}}{l+2}\right)\right)
\end{array}
$$

By Lemma 1 we have, for large $p$ and $l$,

$$
\begin{aligned}
P\left(F_{k, l}^{p}\right) & \leqq \frac{4}{(2 \pi)^{\frac{1}{2}} \psi\left(\frac{2^{p}}{l+2}\right)} e^{-\frac{l}{2(l+2)} \psi^{2}\left(\frac{2 p}{l+2}\right)} \\
& \sim 4 P\left(E_{k, l}^{p}\right) e^{\frac{1}{l+2} \psi^{2}\left(\frac{2^{p}}{l+2}\right)} .
\end{aligned}
$$

Therefore, if $l$ is an integer existing between $\left[\begin{array}{c}p \\ 3\end{array}\right]$ and $p$, there exists a positive constant $c$ such that

$$
P\left(F_{k, l}^{p}\right) \leqq c P\left(E_{k, l}^{p}\right) .
$$

Combining (13) and (16), we obtain

$$
\sum_{p=1}^{\infty} \sum_{k=1}^{2^{p}} \sum_{l=\left[\begin{array}{l}
p \\
3
\end{array}\right]}^{p} P\left(F_{k, l}^{p}\right)<+\infty
$$

According to Borel-Cantelli's lemma in the convergent case, (17) shows that the events $F_{k, l}^{p}$ appearing in (17) occur "only finitely many times" with probability 1 . Or, in other words, there exists a positive $\varepsilon$ with probability 1 such that if $\frac{p}{2^{p+1}}$ is smaller than $\varepsilon, F_{k, l}^{p}$ does not occur for any pair $(k, l)$ appearing in the summation of (17).

Now, for any pair of $\left(t, t^{\prime}\right)$ satisfing the condition $\left|t^{\prime}-t\right|<\varepsilon$, we choose $p$ as follows:

$$
\frac{p+1}{2^{p+1}}<\left|t^{\prime}-t\right| \leqq \frac{p}{2^{p}}<2 \varepsilon .
$$

If we define $k$ and $l$ by the following inequalities

$$
\frac{k-1}{2^{p}}<\min \left(t, t^{\prime}\right) \leqq \frac{k}{2^{p}}<\frac{k+l}{2^{p}} \leqq \max \left(t, t^{\prime}\right)<\frac{k+l+1}{2^{p}},
$$


it follows that $\left[\frac{p}{3}\right]<l \leqq p$ and therefore we obtain

$$
\begin{aligned}
X\left(t^{\prime}\right)-X(t) & \leqq \max _{0 \leqq t, s \leqq \frac{1}{2 p}}\left(X\left(\frac{k+l}{2^{p}}+t\right)-X\left(\frac{k}{2^{p}}-s\right)\right) \\
& \leqq\left(\frac{l}{2^{p}}\right)^{\frac{1}{2}} \psi\left(\frac{2^{p}}{l+2}\right) \\
& \leqq \varphi\left(\left|t^{\prime}-t\right|\right)
\end{aligned}
$$

with probability 1.

Thus Theorem 1 is proved in the convergent case.

2) Proof of the divergent case.

Let $E_{k, l}^{p}$ be the event defined by (12). By the monotony of $\psi(t)$ and Lemma 1 , we have

$$
\begin{aligned}
\sum_{p=1}^{\infty} \sum_{k=1}^{2^{p}} \sum_{l=\left[\begin{array}{l}
p \\
\frac{p}{2}
\end{array}\right]+1}^{p} P\left(E_{k, l}^{p}\right) & =O(1) \sum_{p=1}^{\infty} \sum_{k=1}^{2^{p}} \sum_{l=\left[\frac{p}{2}\right]+1}^{p} e^{-\frac{1}{2} \psi^{2}\left(\frac{2^{p}}{l^{\prime}}\right)} / \psi\left(\frac{2^{p}}{l}\right) \\
& \left.=O(1) \sum_{p=1}^{\infty} \frac{2^{p}}{p} \psi^{3}\left(\frac{2^{p+1}}{p}\right) e^{-\frac{1}{2} \psi^{2}\left(2^{p+1}\right.} \frac{2^{p}}{p}\right) \\
& =O(1) \int^{\infty} \psi^{3}(t) e^{-\frac{1}{2} \psi^{2}(t)} d t=+\infty
\end{aligned}
$$

It is sufficient to show that $E_{k, l}^{p}$ occur "infinitely often" with probability 1. For this purpose, we use the following Lemma given in [4].

LeMMA 2. Let $\left\{E_{k}\right\}$ be a sequence of events satisfying the following conditions.

$$
\sum_{k=1}^{\infty} P\left(E_{k}\right)=+\infty \text {. }
$$

(ii) For every pair of positive integers $h, n$ with $n \geqq h$, there exist $c(h)$ and $H(n, h)$ $>n$ such that for every $m \geqq H(n, h)$ we have

$$
P\left(E_{m} / E_{k}{ }^{\prime}, \cdots, E_{n}{ }^{\prime}\right)>c(h) P\left(E_{m}\right),
$$

where $P(F / E)$ denotes the conditional probability of $F$ on the hypothesis $E$ and $E^{\prime}$ denotes the complement of $E$.

(iii) There exist two absolute constants $c_{1}$ and $c_{2}$ with the following property: to each $E_{j}$ there corresponds a set of events $E_{j_{1}}, \cdots, E_{j_{s}}$ belonging to $\left\{E_{k}\right\}$ such that

$$
\sum_{i=1}^{s} P\left(E_{j} E_{j_{i}}\right)<c_{1} P\left(E_{j_{i}}\right)
$$

and if $k>j$ but $E_{k}$ is not among the $E_{j_{i}}(1 \leqq i \leqq s)$ then

$$
P\left(E_{j} E_{k}\right)<c_{2} P\left(E_{j}\right) P\left(E_{k}\right) \text {. }
$$


Then the probability that the events $E_{k}$ occur "infinitely often" is equal to one.

We rearrange $E_{k, l}^{p}$ and denotes it by $E_{m}$ so that we may apply Lemma 2 in our case. The rule of ordering is given by the following. If $E_{n}=E_{k, t}^{\boldsymbol{p}}$, $E_{m}=E_{k^{\prime}, l}^{p^{\prime}}$, , then $n<m$ if and only if one of the following three conditions holds :

$$
\begin{array}{ll}
(\alpha) & p<p^{\prime}, \\
(\beta) & p=p^{\prime} \quad \text { and } l>l^{\prime},
\end{array}
$$$$
\text { (r) } \quad p=p^{\prime}, \quad l=l^{\prime} \quad \text { and } k<k^{\prime} \text {. }
$$

Now we prove that the sequence $\left\{E_{n}\right\}$ satisfies the conditions of Lemma 2. (i) is a consequence of (20). For (ii), we use the characteristic property of Gaussian distribution. Let $E_{m}=\hat{E}_{k, l}^{p}$ and put $U_{m}=X\left(\frac{k+l}{2^{q}}\right)-X\left(\frac{k}{2^{p}}\right)$. For every pair $(h, n)$ with $n \geqq h$, if we define $U_{h}, U_{h+1}, \cdots, \dot{U}_{n}$ similarly then

$$
\begin{aligned}
& E\left(U_{\imath}\right)=0 \quad(i=h, h+1, \cdots, n), \quad E\left(U_{m}\right)=0, \\
& E\left(U_{i} U_{m}\right) \leqq \frac{l}{2^{p}} \quad(i=h, h+1, \cdots, n),
\end{aligned}
$$

where $E(U)$ denotes the expectation of $U$. Since $\frac{l}{2^{p}}$ tends to zero as $p$ increases, (21) shows that for each $i(h \leqq i \leqq n)$ the correlation coefficient of $U_{i}$ and $U_{m}$ tends to zero as $m$ increases. In other words, $U_{m}$ is asymptotically independent of the joint variable $\left(U_{h}, U_{n+1}, \cdots, U_{n}\right)$. Therefore we have

$$
\lim _{m \rightarrow \infty} \frac{P\left(E_{m} / E_{h}{ }^{\prime}, \cdots, E_{n}{ }^{\prime}\right)}{P\left(E_{m}\right)}=\lim _{m \rightarrow \infty} \frac{P\left(E_{h}{ }^{\prime}, E_{h+1}{ }^{\prime}, \cdots, E_{n}{ }^{\prime} / E_{m}\right)}{P\left(E_{n}{ }^{\prime}, E_{h+1}{ }^{\prime}, \cdots ; E_{n}^{\prime}\right)}=1 .
$$

This shows that (ii) holds in our case. For the justification of (iii), we need some lemmas.

Lemma 3. Let $U$ and $V$ be two random variables whose joint distribution is Gaussian and each of them has a standard Gaussian distribution. Let the correlation coefficient of $U$ and $V$ be $\rho$, then there exists a positive constant $c_{1}$ such that

$$
P(U>a, V>b) \leqq c_{1} P(U>a) P(V>b) \quad \text { for } \rho<\frac{1}{a b} .
$$

$\mathrm{P}_{\mathrm{ROOF}}$. If $\rho$ is negative or if $a$ or $b$ is small, (23) holds trivially. Therefore it is sufficient to prove Lemma 3 for sufficiently large $a, b$ and positive $\rho$. Without loss of generality, we may assume $a \leqq b$. Then we have

$$
P(U>a, V>b)=\frac{1}{2 \pi\left(1-\rho^{2}\right)^{\frac{1}{2}}} \int_{b}^{\infty} \int_{a}^{\infty} e^{-\frac{\left(x^{2}-2 \rho x y+y^{2}\right)}{2\left(1-\rho^{2}\right)}} d x d y
$$


$(24)$

$$
=\frac{1}{2 \pi\left(1-\rho^{2}\right)^{\frac{1}{2}}} \int_{b}^{2 b} \int_{a}^{2 b} e^{-\frac{(x-\rho y)^{2}}{2\left(1-\rho^{2}\right)}} e^{-\frac{y^{2}}{2}} d x d y
$$

$$
\begin{aligned}
& +\frac{1}{2 \pi\left(1-\rho^{2}\right)^{\frac{1}{2}}} \int_{b}^{\infty} \int_{2 b}^{\infty} e^{-\frac{(x-\rho y)^{2}}{2\left(1-\rho^{2}\right)}} e^{-\frac{y^{2}}{2}} d x d y \\
& +\frac{1}{2 \pi\left(1-\rho^{2}\right)^{\frac{1}{2}}} \int_{2 b}^{\infty} \int_{a}^{2 b} e^{-\frac{(x-\rho y)^{2}}{2\left(1-\rho^{2}\right)}} e^{-\frac{y^{2}}{2}} d x d y .
\end{aligned}
$$

The first term on the right side is estimated as follows:

$$
\begin{aligned}
& \frac{1}{2 \pi\left(1-\rho^{2}\right)^{\frac{1}{2}}} \int_{b}^{2 b} \int_{a}^{2 b} e^{-\frac{(x-\rho y)^{2}}{2\left(1-\rho^{2}\right)}} e^{-\frac{y^{2}}{2}} d x d y \\
& \quad \leqq \frac{1}{2 \pi\left(1-\rho^{2}\right)^{\frac{1}{2}}} \int_{b}^{2 b} \int_{a}^{2 b} e^{-\frac{(x-2 / a)^{2}}{2\left(1-\rho^{2}\right)}} e^{-\frac{y^{2}}{2}} d x d y \\
& \quad \leqq P\left\{U>(a-2 / a) /\left(1-\rho^{2}\right)^{\frac{1}{2}}\right\} P(V>b) \\
& \quad=O(1) P(U>a) P(V>b)
\end{aligned}
$$

On the other hand, for sufficiently large $a$, the second and third term on the right side of (24) are trivially smaller than the right side of (23) replaced $c_{1}$ by 1 . These estimates assure the validity of Lemma 3 .

Q.E. D.

Lemma 4. Let $U$ and $V$ be random variables as in Lemma 3 . If the correlation coefficient of $U$ and $V$ is less than $1 / 2^{\frac{1}{2}}$ and $0<a<b$ then there exist two positive constants $c_{2}$ and $\delta_{2}$ satisfying the following inequality

$$
P(U>a, V>b) \leqq c_{2} e^{-\delta_{2} b^{2}} P(U>a) .
$$

Proof. Let $\varepsilon$ be a positive constant which is less than 1 and let $\rho$ be the correlation coefficient of $U$ and $V$. It suffices to prove Lemma 4 for sufficiently large $a$ and positive $\rho$. Then we have

$$
\begin{aligned}
& P(U>a, V>b)=-\frac{1}{2 \pi\left(1-\rho^{2}\right)^{\frac{1}{2}}} \int_{a}^{\infty} \int_{b}^{\infty} e^{-\frac{\left(x^{2}-2 \rho x y+y^{2}\right)}{2\left(1-\rho^{2}\right)}} d x d y \\
& =\frac{1}{2 \pi\left(1-\rho^{2}\right)^{\frac{1}{2}}} \int_{a}^{(1+\varepsilon)^{\frac{1}{2}} b} \int_{b}^{\infty} e^{-\frac{(x-\rho y)^{2}}{2\left(1-\rho^{2}\right)}} e^{-\frac{y^{2}}{2}} d x d y \\
& +\frac{1}{2 \pi\left(1-\rho^{2}\right)^{\frac{1}{2}}} \int_{(1+\varepsilon)^{\frac{1}{2}} \int_{b}}^{\infty} e^{-\frac{(x-\rho y)^{2}}{2\left(1-\rho^{2}\right)}} e^{-\frac{y^{2}}{2}} d x d y
\end{aligned}
$$




$$
\begin{aligned}
\leqq & \frac{1}{2 \pi\left(1-\rho^{2}\right)^{\frac{1}{2}}} \int_{a}^{(1+\varepsilon)^{\frac{1}{2} b}} \int_{b}^{\infty} e^{-\frac{\left(x-(1+\varepsilon / 2)^{\frac{1}{2}}\right) b}{2\left(1-\rho^{2}\right)}} e^{-\frac{y^{2}}{2}} d x d y \\
& +\frac{1}{(2 \pi)^{\frac{1}{2}}} \int_{(1+\varepsilon)^{\frac{1}{2} b}}^{\infty} e^{-\frac{y^{2}}{2}} d y \\
= & O(1)\left\{e^{-\left(1-(1+\varepsilon / 2)^{\frac{1}{2}}\right)^{2 b^{2} / 2}}+e^{-\varepsilon / 2 b^{2}}\right\} P(U>a) .
\end{aligned}
$$

If we take the minimum of $\left(1-(1+\varepsilon / 2)^{\frac{1}{2}}\right)^{2} / 2$ and $\varepsilon / 2$ for $\delta_{2}$ then Lemma 4 follows from (27) immediately.

Q.E. D.

Lemma 5. Let $U$ and $V$ be random variables as in Lemma 3. Denoting the correlation coefficient of $U$ and $V$ by $\rho$, there exist two positive constants $c_{3}$ and $\delta_{3}$ such that

$$
P(U>a, V>a) \leqq c_{3} e^{-\delta_{s}\left(1-\rho^{2}\right) a^{2}} P(U>a) \quad \text { for } a>0 .
$$

Proof. By the definition of Gaussian distribution, we have

$$
P(U>a, V>a)=\frac{1}{2 \pi\left(1-\rho^{2}\right)^{\frac{1}{2}}} \int_{a}^{\infty} \int_{a}^{\infty} e^{-\frac{\left(x^{2}-2 \rho x y+y^{2}\right)}{2\left(1-\rho^{2}\right)}} d y d x .
$$

Rotating the axes by $\pi / 4$, we obtain

$$
\begin{aligned}
P(U>a, V>a) & =\frac{1}{2 \pi\left(1-\rho^{2}\right)^{\frac{1}{2}}} \int_{2^{\frac{1}{2}} a}^{\infty} \int_{-\left(x-2^{\frac{1}{2}} a\right)}^{\left(x+2^{\frac{1}{2}} a\right)} e^{-\frac{(1-\rho) x^{2}+(1+\rho) y^{2}}{2\left(1-\rho^{2}\right)}} d y d x \\
& \leqq \frac{1}{(2 \pi)^{\frac{1}{2}}} \int_{(2 / 1+\rho)^{\frac{1}{2}} a}^{\infty} e^{-\frac{x^{2}}{2}} d x \\
& =O(1) e^{-\frac{1-\rho^{2}}{2(1+\rho)^{2}} a^{2}} \cdot \frac{1}{a} e^{-\frac{a^{2}}{2}} \\
& =O(1) e^{-\frac{1-\rho^{2}}{2(1+\rho)^{2}}} P(U>a) .
\end{aligned}
$$

If we take $1 / 8$ for $\delta_{3}$, Lemma 5 follows from (31).

Q.E.D.

Now we prove that the condition (iii) of Lemma 2 is satisfied by our sequence $\left\{E_{n}\right\}$. For given $E_{j}$, recalling that $E_{j}$ has another expression $E_{k, l}^{p}$, we choose a sequence $\left\{E_{j i} ; i=1,2, \cdots, s\right\}$ of events with the properties that $j_{i}>j$, the corresponding superscript $p^{\prime}$ is less than $(p+5 \log p)$ and $E_{j_{i}}$ is not independent of $E_{j}$. If $E_{m}$ is independent of $E_{j}$ then (b) of (ii) holds trivially for $c_{2}=1$. On the other hand, if $E_{m}$ is not independent of $E_{j}$, we use Lemma 3. Let $E_{j}=E_{k, l}^{p}$ and $E_{m}=E_{k^{\prime}, l^{\prime}}^{p^{\prime}}$. If $m$ is not one of the $j_{i}^{\prime}$ s then it follows from the definition of $\left\{E_{j_{i}}\right\}$ that $(p+5 \log p)<p^{\prime}$. Considering only the case of $l>\frac{p}{2}$, we have by Lemma 1 and for large $p$, 


$$
\begin{aligned}
E\left\{\frac{\left(X\left(\frac{k+l}{2^{p}}\right)-X\left(\frac{k}{2^{p}}\right)\right)}{\left(\frac{l}{2^{p}}\right)^{\frac{1}{2}}} \cdot \frac{\left(X\left(\frac{k^{\prime}+l^{\prime}}{2^{p^{\prime}}}\right)-X\left(\frac{k^{\prime}}{2^{p^{\prime}}}\right)\right)}{\left(\frac{l^{\prime}}{2^{p^{\prime}}}\right)^{\frac{1}{2}}}\right\} & \leqq\left(\frac{p^{\prime}}{2^{p^{\prime}}}\right)^{\frac{1}{2}}\left(\frac{2^{p+1}}{p}\right)^{\frac{1}{2}} \\
& \leqq \frac{1}{\psi\left(\frac{2^{p}}{p}\right) \psi\left(\frac{2^{p^{\prime}}}{p^{\prime}}\right)}
\end{aligned}
$$

Since the joint distribution of the two random variables appearing in (30) is a Gaussian distribution in 2-dimension's, we may use Lemma 3, Thus there exists a positive constant $c$ such that

$$
P\left(E_{j} E_{m}\right)=P\left\{X\left(\frac{k+l}{2^{p}}\right)-X\left(\frac{k}{2^{p}}\right)>\varphi\left(\frac{l}{2^{p}}\right), X\left(\frac{k^{\prime}+l^{\prime}}{2^{p^{\prime}}}\right)-X\left(\frac{k^{\prime}}{2^{p^{\prime}}}\right)>\varphi\left(\frac{l^{\prime}}{2^{p^{\prime}}}\right)\right\}
$$

$$
\leqq c P\left(E_{j}\right) P\left(E_{m}\right) \text {. }
$$

If we take the maximum of $c$ and 1 for $c_{2}$ in (b) of (iii) then (b) holds.

In order to verify (a) of (iii), we use the other expressions of the $E_{j}$ 's. Let us denote $E_{j}$ by $E_{k, l}^{p}$ and each one of $E_{j_{i}}$ by $E_{k, l}^{p}$. Dividing the sum of $P\left(E_{j} E_{j_{i}}\right)$ according to the magnitude of the correlation coefficient of $\left(X\left(\frac{k+l}{2^{p}}\right)-X\left(\frac{k}{2^{p}}\right)\right)$ and $\left(X\left(\frac{k^{\prime}+l^{\prime}}{2^{p^{\prime}}}\right)-X\left(\frac{k^{\prime}}{2^{p^{\prime}}}\right)\right)$ we have

$$
\sum_{i=1}^{s} P\left(E_{j} E_{j_{i}}\right)=\Sigma^{\prime} P\left(E_{j} E_{j_{i}}\right)+\Sigma^{\prime \prime} P\left(E_{j} E_{j_{i}}\right),
$$

where $\Sigma^{\prime}$ denotes the summation over $i$ 's such that the correlation coefficient of the corresponding random variables is larger than $\frac{1}{\sqrt{2}}$ and $\Sigma^{\prime \prime}$ denotes the summation of the remainder. Since the correlation is at most

$$
\min \left(\frac{l}{2^{p}}, \frac{l^{\prime}}{2^{p^{\prime}}}\right)\left(\frac{l l^{\prime}}{2^{p+p^{\prime}}}\right)^{-1 / 2}
$$

and since $l^{\prime} 2^{-p^{\prime}} \leqq l 2^{-p}$ by the limitation on the ranges of $l$ and $l^{\prime}$, we see that the largest superscript of $E_{j i}$ 's appearing in $\Sigma^{\prime}$ is at most $p+2$. Moreover, without loss of generality, we may assume in the computation of $P\left(E_{j} E_{j_{i}}\right)$ that $\frac{k}{2^{p}} \leqq \frac{k^{\prime}}{2^{p^{\prime}}}$. If $\frac{k+l}{2^{p}} \leqq \frac{k^{\prime}+l^{\prime}}{2^{p^{\prime}}}$, we have

$$
P\left(E_{j} E_{j_{i}}\right)=P\left(\frac{X\left(\frac{k+l}{2^{p}}\right)-X\left(\frac{k}{2^{p}}\right)}{\left(\frac{l}{2^{p}}\right)^{\frac{1}{2}}}>\psi\left(\frac{2^{p}}{l}\right), \frac{X\left(\frac{k^{\prime}+l^{\prime}}{2^{p^{\prime}}}\right)-X\left(\frac{k^{\prime}}{2^{p^{\prime}}}\right)}{\left(\frac{l^{\prime}}{2^{p^{\prime}}}\right)^{\frac{1}{2}}}>\psi\left(\frac{2^{p^{\prime}}}{l^{\prime}}\right)\right)
$$

$$
\leqq P\left(\frac{X\left(\frac{k+l}{2^{p}}\right)-X\left(\frac{k}{2^{p}}\right)}{\left(\frac{l}{2^{p}}\right)^{\frac{1}{2}}}>\psi\left(\frac{2^{p}}{l}\right), \frac{X\left(\frac{k+l}{2^{p}}\right)-X\left(\frac{k^{\prime}}{2^{p^{\prime}}}\right)}{\left(\frac{k+l}{2^{p}}-\frac{k^{\prime}}{2^{p^{\prime}}}\right)^{\frac{1}{2}}}>\psi\left(\frac{2^{p}}{l}\right)\right)
$$


The inequality follows from the definition of ordering and the fact that the correlation coefficient of two random variables appearing in the last term of (33) is larger than that. of the second term. Since $p^{\prime} \leqq p+2$, we obtain by Lemma 1 and Lemma 5 that

$$
P\left(E_{j} E_{j_{i}}\right) \leqq c e^{-\delta\left(\frac{\left(k^{\prime}-k 2^{p^{\prime}-p}\right.}{2} 2^{p^{\prime}-p}\right) \psi^{2}\left(\frac{2^{p}}{l}\right)} P\left(E_{j}\right)
$$

$$
\leqq c e^{-\delta^{\prime}\left(k^{\prime}-k 2^{\left.p^{\prime}-p\right)}\right.} P\left(E_{j}\right),
$$

where $c, \delta$ and $\delta^{\prime}$ are positive constants. Here we remark that the number of $E_{j_{i}}$ appearing in the present case is less than $\left(k^{\prime}-k 2^{p /-p}\right)$ for fixed pair $\left(p^{\prime}, k^{\prime}\right)$ because $\frac{l}{2^{p}} \geqq \frac{l^{\prime}}{2^{p^{\prime}}}$. Similarly, for the case of $\frac{k}{2^{p}}>\frac{k^{\prime}}{2^{p^{\prime}}}$ we have

$$
P\left(E_{j} E_{j_{i}}\right) \leqq c e^{-\delta^{\prime}\left(l 2^{p^{\prime}-p_{\left.-l^{\prime}\right)}}\right.} P\left(E_{j}\right) .
$$

Considering the same situation for $\frac{k}{2^{p}}>\frac{k^{\prime}}{2^{p^{\prime}}}$, we have

$$
\begin{aligned}
\Sigma^{\prime} P\left(E_{j} E_{j_{i}}\right) \leqq 2 c P\left(E_{j}\right) \sum_{p^{\prime}=p}^{p+2}\{ & \left\{\sum_{k^{\prime}=k 2^{p^{\prime}-p}}^{(k+l) 2^{p^{\prime}-p}}\left(k^{\prime}-k 2^{p^{\prime}-p}\right) e^{-\delta^{\prime}\left(k^{\prime}-k 2^{p^{\prime}}-p\right.}\right) \\
& \left.+\sum_{l^{\prime}=1}^{\iota 2^{p^{\prime}-p}}\left(l 2^{p^{\prime}-p}-l^{\prime}\right) e^{-\delta\left(l^{p^{p^{\prime}}-p_{-}}-l^{\prime}\right)}\right\} \\
\leqq &
\end{aligned}
$$

where $\alpha$ is an absolute constant.

For the computation of $P\left(E_{j} E_{j_{i}}\right)$ where $E_{j_{i}}$ appears in the summation of $\Sigma^{\prime \prime}$, we apply Lemma 4. Using the same expression for $E_{i}$ and $E_{j_{i}}$ as before, for the case of $\frac{k}{2^{p}} \leqq \frac{k^{\prime}}{2^{p^{\prime}}}<\frac{k+l}{2^{p}} \leqq \frac{k^{\prime}+l^{\prime}}{2^{p^{\prime}}}$, we have

$$
\begin{aligned}
P\left(E_{j} E_{j_{i}}\right) & \leqq P\left(\frac{X\left(\frac{k+l}{2^{p}}\right)-X\left(\frac{k}{2^{p}}\right)}{\left(\frac{l}{2^{p}}\right)^{\frac{1}{2}}}>\psi\left(\frac{2^{p}}{l}\right), \frac{X\left(\frac{k+l}{2^{p}}\right)-X\left(\frac{k^{\prime}}{2^{p^{\prime}}}\right)}{\left(\frac{k+l}{2^{p}}-\frac{k^{\prime}}{2^{p^{\prime}}}\right)^{\frac{1}{2}}}>\psi\left(\frac{2^{p^{\prime}}}{l^{\prime}}\right)\right) \\
& \leqq c e^{-\delta \psi^{2}\left(\frac{2^{p^{\prime}}}{l^{\prime}}\right)} P\left(E_{j}\right) \\
& \leqq c e^{-\delta^{\prime} p^{\prime}} P\left(E_{j}\right)
\end{aligned}
$$

where $c, c^{\prime}, \delta$ and $\delta^{\prime}$ are positive constants. Similarly, for the case of $\frac{k}{2^{p}}$ $\leqq \frac{k^{\prime}}{2^{p^{\prime}}}<\frac{k^{\prime}+l^{\prime}}{2^{p^{\prime}}} \leqq \frac{k+l}{2^{p}}$, we obtain the same result. Combining all the cases, we have 


$$
\begin{aligned}
\Sigma^{\prime \prime} P\left(E_{j} E_{j i}\right) & \leqq c P\left(E_{j}\right) \sum_{p^{\prime}=p}^{p+5 \log p} p^{\prime 2} 2^{p^{\prime}-p} l e^{-\delta^{\prime} p^{\prime}} \\
& \leqq c P\left(E_{j}\right) \sum_{p^{\prime}=p}^{p+5 \log p} p^{\prime 8} e^{-\delta^{\prime} p^{\prime}} \\
& =\beta P\left(E_{j}\right),
\end{aligned}
$$

where $\beta$ is an absolute constant. (32), (36) and (38) establish the validity of (iii a). Therefore we may aplly Lemma 2 in our case and Theorem 1 is proved completely.

Q.E. D.

Syracuse University, Technion, Israel, and Shizuoka University.

\section{References}

[1] P. Lévy, Théorie de l'addition des variables aléatoires, 1937.

[2] T. Sirao, On the uniform continuity of Wiener process, J. Math. Soc. Japan, 6, (1954), 332-335.

[3] P. Lévy, Le mouvement brownien, Mém. Sci. Math., fasc. 126, 1954.

[4] K.L. Chung and P. Erdös, On the application of the Borel-Cantelli Lemma, Trans. Amer. Math. Soc., 72, (1952), 179-189. 